\title{
Lyapunov Exponents for Unitary Anderson Models
}

\author{
Eman Hamza and Günter Stolz* \\ University of Alabama at Birmingham \\ Department of Mathematics CH 452 \\ Birmingham, AL 35294-1170 \\ U.S.A.
}

\begin{abstract}
We study a unitary version of the one-dimensional Anderson model, given by a five diagonal deterministic unitary operator multiplicatively perturbed by a random phase matrix. We fully characterize positivity and vanishing of the Lyapunov exponent for this model throughout the spectrum and for arbitrary distributions of the random phases. This includes Bernoulli distributions, where in certain cases a finite number of critical spectral values, with vanishing Lyapunov exponent, exists. We establish similar results for a unitary version of the random dimer model.
\end{abstract}

\section{Introduction}

Unitary operators arise naturally in quantum mechanics as the time evolution of the Hamiltonian in solving the time dependent Schrödinger equation. In particular, for Hamiltonians with periodically time-dependent potentials, the spectral properties of the monodromy operator (the unitary operator giving the evolution over one time period) are the central object of mathematical investigations (e.g. 1], 9], 14]). A model using random unitary operators was used in [3] to study the single particle behavior of an electron in a small one-dimensional metal ring in the presence of a large uniform electric field generated by a linearly ramped magnetic flux. The unitary operator used in describing this system is characterized by a five diagonal band structure. Motivated by this model, the spectral analysis of a class of random unitary operators with similar band structure was undertaken in [4], [16], 18] and [13.

This class of operators on $l^{2}(\mathbb{Z})$ can be written (up to a unitary equivalence) in the form

$$
U_{\omega}=D_{\omega} S
$$

where $S$ is a unitary operator with a five-diagonal matrix representation. $S$ depends on a parameter $t \in(0,1)$ which controls the size of its off-diagonal elements and takes the role of a disorder parameter for $U_{\omega}$, see Section 2 below. $D_{\omega}$ is a diagonal matrix of random phases, $\operatorname{diag}\left\{e^{-i \theta_{k}^{\omega}}\right\}$. For our application, $\left\{\theta_{k}^{\omega}: k \in \mathbb{Z}\right\}$ is a sequence of i.i.d. random variables on the

*partially supported through US-NSF grant DMS-0245210 
one-dimensional torus $\mathbb{T}=\mathbb{R} / 2 \pi \mathbb{Z}$ with a non-trivial probability distribution $\mu$, i.e. $\operatorname{supp} \mu$ has two or more elements. $U_{\omega}$ can be considered as a "unitary Anderson-type model", where $S$ plays the role of the free Laplacian and where the perturbation is introduced via multiplication rather than addition to ensure that the resulting operator is still unitary. Indeed, due to the band structure of the operator, the generalized eigenvectors can be studied using complex $2 \times 2$ transfer matrices. This formalism allows to introduce the Lyapunov exponent $\gamma(\lambda)$, where $e^{i \lambda}$ is the spectral parameter, see equation (8) below. Due to the fact that the transfer matrices have determinants of unit modulus, the Lyapunov exponent is almost surely non-negative. This also allowed to prove a unitary version of the Ishii-Pastur Theorem, i.e. Theorem 2.1 below, and to deduce the absence of absolutely continuous spectrum in case of uniform distribution $\mu$ [4]. In [13] it was shown that the spectrum of $U_{\omega}$ is almost surely pure point with exponentially decaying eigenfunctions if the distribution $\mu$ has a non-trivial absolutely continuous component and non-empty interior. This holds for arbitrary value of the disorder parameter $t$. Thus, up to this point, the results for one-dimensional unitary Anderson models are in close analogy to those known for the self-adjoint Anderson model.

Further results on unitary Anderson models were found in [16], where the density of states is studied, and in [18, which develops a fractional moment approach to prove localization for multi-dimensional analogs of unitary Anderson models.

The main goal of this paper is to further investigate the model (1) and to fully determine the set $\{\lambda \in \mathbb{T}: \gamma(\lambda)>0\}$ for arbitrary disorder $t$ and arbitrary distribution $\mu$, including singular and, in particular, Bernoulli distributions.

For most choices of $\mu$ we find that $\gamma(\lambda)>0$ for all "quasi-energies" $\lambda \in \mathbb{T}$, see Theorem 3.2. However, there is one exceptional situation: If $\mu$ is a Bernoulli measure supported on two diametrically opposed points, i.e. $\operatorname{supp} \mu=\{a, b\},|a-b|=\pi$, then there exist two critical quasi-energies $\lambda=-a$ and $\lambda=-b$ at which the Lyapunov exponent vanishes, while it is positive for all other values of $\lambda$ (Theorem 3.1). In fact, we show in the proof of Theorem 3.1 that at the anomalies $\lambda=-a$ and $\lambda=-b$ the transfer matrices $T_{n}(\omega, \lambda)$ satisfy the asymptotics

$$
\frac{1}{n} \mathbb{E}\left(\left(\ln \left\|T_{n}(\omega, \lambda)\right\|\right)^{2}\right) \longrightarrow C>0,
$$

i.e., roughly, $\left\|T_{n}(\omega, \lambda)\right\| \sim e^{(C n)^{1 / 2}}$.

As there are no more than two critical values of $\lambda$, the unitary version of the IshiiPastur Theorem shows that the unitary Anderson model (1) almost surely has no absolutely continuous spectrum, irrespective of the underlying probability measure and disorder. We expect that methods such as those used in [8] can be adjusted to also show that $U_{\omega}$ almost surely has pure point spectrum with exponentially decaying eigenfunctions, i.e. is spectrally localized, but we haven't carried out the details of this.

It is interesting that the structure of Lyapunov exponents for the unitary Anderson model is richer than for the self-adjoint one-dimensional Anderson model. For the latter it has been long known that the Lyapunov exponent is positive at all energies for all nontrivial single site distributions of the random potential, e.g. [5].

In a more general class of self-adjoint Anderson-type models it has been shown that the existence of critical energies with vanishing Lyapunov exponents can lead to the coexistence of spectral localization and suitable forms of dynamical delocalization, e.g. [15]. The simplest self-adjoint model which shows this phenomenon is the so-called dimer model [12, 11], in which the random phases appear in the form of identical neighboring pairs. The 
typical anomalies encountered in the dimer model are stronger than those in Theorem 3.1 below in the sense that transfer matrices, rather than satisfying (2), are uniformly bounded in $n$. In [15] it is shown that this leads to super-diffusive transport, while, to our knowledge, the dynamical effects caused by an anomaly as in (2) have not been studied (they should be much weaker, if detectable at all).

In Section [6 we study a unitary version of the dimer model, where we can show that the Lyapunov exponent is positive away from at most finitely many critical values. However, for the dimer model with Bernoulli distributed phases, i.e. $\operatorname{supp} \mu=\{a, b\}$, and such that $|a-b|$ is in the spectrum of $S$, there are two critical values where transfer matrices are of the type studied in [15], in particular they are bounded in $n$.

While we do not carry out a complete study of spectral and dynamical localization properties of unitary Anderson models, in Section 7 we state one more result which has been relevant in this context in the self-adjoint case, namely continuity of the Lyapunov exponent in $\lambda$ away from the critical quasi-energies. This is proven by a rather direct adaptation of the proof in the self-adjoint case, e.g. [5].

Let us finally mention that unitary operators with the same band structure as $S$ and $U_{\omega}$ above also arise in the form of so-called CMV-matrices in the study of orthogonal polynomials on the unit circle, e.g. [19, 6]. In this setting, a definition of Lyapunov exponents different from ours arises naturally (applicable for example for the case of i.i.d. random Verblunsky coefficients), see Section 10.5 in [19]. However, it can be shown that both definitions lead to the same value [17] (if either one of the two Lyapunov exponents exists).

\section{The Model}

Analogous to the self-adjoint case, we look at a random unitary operator as a random perturbation of a deterministic ("free") unitary operator. The model and the results presented in this section can be found in [4, [13, [16] and [18, see there for details and additional motivation and results. Motivated by [3], we choose the free unitary operator $S$ on $l^{2}(\mathbb{Z})$ with band structure

$$
S=\left(\begin{array}{cccccc}
\ddots & r t & -t^{2} & & & \\
& r^{2} & -r t & & & \\
& r t & r^{2} & r t & -t^{2} & \\
& -t^{2} & -t r & r^{2} & -r t & \\
& & & r t & r^{2} & \\
& & & -t^{2} & -t r & \ddots
\end{array}\right)
$$

where the position of the origin in $\mathbb{Z}$ is fixed by $\left\langle e_{2 k-2}, S e_{2 k}\right\rangle=-t^{2}$, with $e_{k}(k \in \mathbb{Z})$ denoting the canonical basis vectors in $l^{2}(\mathbb{Z})$. The real parameters $t$ and $r$ are linked by $r^{2}+t^{2}=1$ to ensure unitarity. Due to unitary equivalence it suffices to consider $0 \leq t, r \leq 1$. Thus $S$ is determined by $t$. We shall sometimes write $S(t)$ to emphasize this dependence. Excluding trivial special cases, we assume $0<t<1$. The spectrum of $S(t)$ is purely absolutely continuous and is given by the arc

$$
\sigma(S(t))=\Sigma(t)=\left\{e^{i \vartheta}: \vartheta \in\left[-\arccos \left(1-2 t^{2}\right), \arccos \left(1-2 t^{2}\right)\right]\right\}
$$

which is symmetric about the real axis and grows from the single point $\{1\}$ for $t=0$ to the entire unit circle for $t=1$. 
The random perturbation is then introduced via multiplication by a diagonal matrix

$$
D_{\omega}=\operatorname{diag}\left\{e^{-i \theta_{k}^{\omega}}\right\}
$$

with $\left\{\theta_{k}^{\omega}: k \in \mathbb{Z}\right\}$ a sequence of i.i.d. random variables on the torus $\mathbb{T}=\mathbb{R} / 2 \pi \mathbb{Z}$. More precisely, we introduce the probability space $(\Omega, \mathcal{F}, \mathbb{P})$, where $\Omega$ is identified with $\mathbb{T}^{\mathbb{Z}}, \mathcal{F}$ is the $\sigma$-algebra generated by cylinders of Borel sets, and $\mathbb{P}=\bigotimes_{k \in \mathbb{Z}} \mu$, where $\mu$ is a probability measure on $\mathbb{T}$. The random variables $\theta_{k}$ on $(\Omega, \mathcal{F}, \mathbb{P})$ are defined by

$$
\theta_{k}: \Omega \rightarrow \mathbb{T}, \quad \theta_{k}^{\omega}=\omega_{k}, \quad k \in \mathbb{Z}
$$

This ensures that the resulting operator

$$
U_{\omega}=D_{\omega} S
$$

is unitary and ergodic with respect to the 2-shift in $\Omega$ [4]. $U_{\omega}$ also inherits the band structure of the original operator $S$ and has the almost sure spectrum [16]

$$
\Sigma=\exp (i \operatorname{supp} \mu) \Sigma(t)=\left\{e^{i \alpha} \Sigma(t) \mid \alpha \in \operatorname{supp} \mu\right\} .
$$

Here supp $\mu$ denotes the support of the probability measure $\mu$, defined as

$$
\operatorname{supp} \mu:=\{a \mid \mu(a-\epsilon, a+\epsilon)>0 \text { for all } \epsilon>0\} .
$$

Solutions of the eigenvalue equation

$$
U_{\omega} \psi=e^{i \lambda} \psi, \quad \psi=\sum_{k \in \mathbb{Z}} c_{k} e_{k}
$$

with $c_{k} \in \mathbb{C}, \lambda \in \mathbb{C}$, are characterized by the relations

$$
\left(\begin{array}{c}
c_{2 k+1} \\
c_{2 k+2}
\end{array}\right)=T\left(\theta_{2 k}^{\omega}(\lambda), \theta_{2 k+1}^{\omega}(\lambda)\right)\left(\begin{array}{c}
c_{2 k-1} \\
c_{2 k}
\end{array}\right)
$$

for all $k \in \mathbb{Z}$, where the transfer matrices $T: \mathbb{T}^{2} \rightarrow G L(2, \mathbb{C})$ are defined by

$$
T(\theta, \eta)=\left(\begin{array}{cc}
-e^{-i \eta} & \frac{r}{t}\left(e^{i(\theta-\eta)}-e^{-i \eta}\right) \\
\frac{r}{t}\left(1-e^{-i \eta}\right) & -\frac{1}{t^{2}} e^{i \theta}+\frac{r^{2}}{t^{2}}\left(1+e^{i(\theta-\eta)}-e^{-i \eta}\right)
\end{array}\right),
$$

and the phases by

$$
\theta_{k}^{\omega}(\lambda)=\theta_{k}^{\omega}+\lambda
$$

Note that $\operatorname{det} T\left(\theta_{2 k}^{\omega}(\lambda), \theta_{2 k+1}^{\omega}(\lambda)\right)=e^{i\left(\theta_{2 k}^{\omega}-\theta_{2 k+1}^{\omega}\right)}$ has modulus one and is independent of $\lambda$.

We have for any $n \in \mathbb{N}$

$$
\begin{aligned}
\left(\begin{array}{c}
c_{2 n-1} \\
c_{2 n}
\end{array}\right) & =T\left(\theta_{2(n-1)}^{\omega}(\lambda), \theta_{2(n-1)+1}^{\omega}(\lambda)\right) \cdots T\left(\theta_{0}^{\omega}(\lambda), \theta_{1}^{\omega}(\lambda)\right)\left(\begin{array}{c}
c_{-1} \\
c_{0}
\end{array}\right) \equiv T_{n}(\omega, \lambda)\left(\begin{array}{c}
c_{-1} \\
c_{0}
\end{array}\right) \\
\left(\begin{array}{c}
c_{-2 n-1} \\
c_{-2 n}
\end{array}\right) & =T\left(\theta_{-2 n}^{\omega}(\lambda), \theta_{-2 n+1}^{\omega}(\lambda)\right)^{-1} \cdots T\left(\theta_{-2}^{\omega}(\lambda), \theta_{-1}^{\omega}(\lambda)\right)^{-1}\left(\begin{array}{c}
c_{-1} \\
c_{0}
\end{array}\right) \equiv T_{-n}(\omega, \lambda)\left(\begin{array}{c}
c_{-1} \\
c_{0}
\end{array}\right) .
\end{aligned}
$$

We also set $T_{0}(\omega, \lambda)=\mathbb{I}$. 
As shown in [4], for any $\lambda \in \mathbb{C}$, the Lyapunov exponent

$$
\gamma_{\omega}^{ \pm}(\lambda)=\lim _{n \rightarrow \pm \infty} \frac{1}{|n|} \ln \left\|T_{n}(\omega, \lambda)\right\|
$$

almost surely exists, has the same value for $k \rightarrow \infty$ and $k \rightarrow-\infty$, and takes the deterministic value

$$
\gamma(\lambda)=\lim _{n \rightarrow \infty} \frac{\mathbb{E}\left(\ln \left\|T_{n}(\omega, \lambda)\right\|\right)}{n} .
$$

A version of the Ishii-Pastur theorem suited to the present model was proven in [4].

Theorem 2.1. Let $U_{\omega}$ be defined by (6), (4) and (5) and $\gamma(\lambda)$ by (8). Then

$$
\Sigma_{a c} \subseteq{\overline{\left\{e^{i \lambda} \in S^{1} ; \gamma(\lambda)=0\right\}}}^{\text {ess }} .
$$

All norms on $G L(n, \mathbb{C})$ being equivalent, we choose to work with the row-sum norm for convenience. Thus, in what follows the norm is the maximum row sum, i.e. for $A=$ $\left(a_{i j}\right)_{i, j=1}^{n},\|A\|=\max _{1 \leq i \leq n} \sum_{j=1}^{n}\left|a_{i j}\right| . \mathbf{P}\left(\mathbb{C}^{2}\right)$ denotes the projective space of $\mathbb{C}^{2}$, we write $\bar{v} \in \mathbf{P}\left(\mathbb{C}^{2}\right)$ for the direction of $v \in \mathbb{C}^{2} \backslash\{0\}$. The action of a $2 \times 2$ matrix $A$ on $\mathbf{P}\left(\mathbb{C}^{2}\right)$ is defined by $A \bar{v}=\overline{A v}$.

\section{The Main Results}

For a particular choice of the underlying distribution of the random phases the unitary Anderson model, unlike the self-adjoint one, exhibits two critical values of the spectral parameter, where the Lyapunov exponent vanishes.

Theorem 3.1. If supp $\mu=\{a, b\}$ and $|a-b|=\pi$, then

(i) $\gamma(-a)=\gamma(-b)=0$.

(ii) $\gamma(\lambda)>0$, for all $\lambda \in \mathbb{T} \backslash\{-a,-b\}$.

This is the only exceptional case. For all other choices of the probability measure $\mu$ the Lyapunov exponent never vanishes.

Theorem 3.2. If $\{a, b\} \subset$ supp $\mu$ such that $|a-b| \notin\{0, \pi\}$, then for every $\lambda \in \mathbb{T}$ we have $\gamma(\lambda)>0$. In particular, if supp $\mu$ contains at least three elements, then $\gamma(\lambda)>0$ for all $\lambda \in \mathbb{T}$.

Theorem 3.2 is a generalization of the corresponding results on the Lyapunov exponent previously proven in [4] and [13].

Theorem [3.1(i) will be proven in Section 4, while the proofs of Theorem 3.1(ii) and Theorem 3.2 are provided in Section 5 .

The fact that for all non-trivial probability measures $\mu$, the set of critical quasi-energies contains at most two points combined with Theorem 2.1 gives the following immediate corollary, concerning the almost sure absolutely continuous spectrum of $U_{\omega}$.

Corollary 3.1. For any non-trivial distribution $\mu$ of the i.i.d. random phases, we have

$$
\Sigma_{a c}=\emptyset .
$$




\section{Critical Quasi-energies}

In this section we prove Theorem 3.1(i), i.e. that a Bernoulli measure $\mu$ with diametrically opposed masses $a, b$ indeed gives rise to two critical quasi-energies at $\lambda=-a, \lambda=-b$. Denote $\mu(a)=p \in(0,1)$ and $\mu(b)=q=1-p$.

For $\lambda=-a$, the i.i.d. random matrices $T\left(\theta_{2 k}^{\omega}(\lambda), \theta_{2 k+1}^{\omega}(\lambda)\right)$ take only the following values with non-zero probabilities,

$$
T\left(\theta_{2 k}^{\omega}(\lambda), \theta_{2 k+1}^{\omega}(\lambda)\right)= \begin{cases}T(0,0)=-I, & \text { with probability } p^{2} \\
T(\pi, \pi)=\left(\begin{array}{cc}
1 & 2 r / t \\
2 r / t & \left(3 r^{2}+1\right) / t^{2}
\end{array}\right), & \text { with probability } q^{2} \\
T(\pi, 0)=\left(\begin{array}{cc}
-1 & -2 r / t \\
0 & 1
\end{array}\right), & \text { with probability } p q \\
T(0, \pi)=\left(\begin{array}{cc}
1 & 0 \\
2 r / t & -1
\end{array}\right), & \text { with probability } p q .\end{cases}
$$

The latter matrices take much simpler forms when represented with respect to the basis

$\left\{\left(\begin{array}{c}1 \\ (r+1) / t\end{array}\right),\left(\begin{array}{c}1 \\ (r-1) / t\end{array}\right)\right\}$ of $\mathbb{C}^{2}$. Hence, we define the matrices $A(\theta, \eta)$ as

$$
A(\theta, \eta):=\left(\begin{array}{cc}
1 & 1 \\
(r+1) / t & (r-1) / t
\end{array}\right)^{-1} T(\theta, \eta)\left(\begin{array}{cc}
1 & 1 \\
(r+1) / t & (r-1) / t
\end{array}\right) .
$$

It follows that

$$
\begin{aligned}
A(0,0)=-I, & A(\pi, \pi)=\left(\begin{array}{cc}
\rho & 0 \\
0 & 1 / \rho
\end{array}\right) \\
A(\pi, 0)=\left(\begin{array}{cc}
0 & -1 / \rho \\
-\rho & 0
\end{array}\right), & A(0, \pi)=\left(\begin{array}{ll}
0 & 1 \\
1 & 0
\end{array}\right),
\end{aligned}
$$

with $\rho:=(r+1)^{2} / t^{2}>1$.

A straightforward calculation shows that

$$
\gamma(\lambda)=\lim _{n \rightarrow \infty} \frac{\mathbb{E}\left(\ln \left\|\Lambda_{n}(\omega, \lambda)\right\|\right)}{n},
$$

where $\Lambda_{n}(\omega, \lambda)=\Pi_{k=1}^{n} A\left(\theta_{2 k}^{\omega}(\lambda), \theta_{2 k+1}^{\omega}(\lambda)\right)$. In order to simplify the notation, we will suppress the $\omega$ dependence of various quantities for the remainder of the section.

Let $u_{0}:=\left(\begin{array}{l}1 \\ 1\end{array}\right)$ and $u_{n}(\lambda)=\left(\begin{array}{l}u_{n, 1}(\lambda) \\ u_{n, 2}(\lambda)\end{array}\right):=\Lambda_{n}(\lambda) u_{0}$.

Lemma 4.1. If $x_{n}=\frac{\ln \left|u_{n, 1}(\lambda)\right|}{\ln \rho}$ for $n \geq 0$, then

$$
\gamma(-a)=\ln \rho \lim _{n \rightarrow \infty} \frac{1}{n} \mathbb{E}\left(\left|x_{n}\right|\right) .
$$

Proof. From (10) it follows that with probability one there are $x, y \in \mathbb{R}$ with $x y=1$ and either $\Lambda_{n}(-a)=\left(\begin{array}{ll}x & 0 \\ 0 & y\end{array}\right)$ or $\Lambda_{n}(-a)=\left(\begin{array}{ll}0 & x \\ y & 0\end{array}\right)$. In both cases it follows readily that

$$
\left\|\Lambda_{n}(-a)\right\|=\left\|\Lambda_{n}(-a) u_{0}\right\|_{\infty},
$$


where $\|\cdot\|_{\infty}$ denotes the max-norm on $\mathbb{C}^{2}$. This implies that

$$
\gamma(-a)=\lim _{n \rightarrow \infty} \frac{1}{n} \mathbb{E}\left(\ln \left\|\Lambda_{n}(-a) u_{0}\right\|_{\infty}\right)
$$

Furthermore we see from the specific form of $\Lambda_{n}(-a)$ that $\Lambda_{n}(-a) u_{0}=\left(\begin{array}{c}u_{n, 1}(-a) \\ 1 / u_{n, 1}(-a)\end{array}\right)$. Therefore

$$
\begin{aligned}
\ln \left\|\Lambda_{n}(-a) u_{0}\right\|_{\infty} & =\ln \max \left(\left|u_{n, 1}(-a)\right|, \frac{1}{\left|u_{n, 1}(-a)\right|}\right) \\
& =|\ln | u_{n, 1}(-a)||
\end{aligned}
$$

The required result then follows from (13) and the definition of $x_{n}$.

The following lemma is devoted to the necessary analysis of the random sequence $x_{n}$.

Lemma 4.2. $\left(x_{n}\right)_{n \geq 0}$ is an integer-valued Markov chain with $x_{0}=0$ and transition probabilities

$$
\begin{aligned}
\mathbb{P}\left(x_{n+1}=x_{n}\right) & =p^{2}, & & \mathbb{P}\left(x_{n+1}=x_{n}+1\right)=q^{2}, \\
\mathbb{P}\left(x_{n+1}=-x_{n}\right) & =p q, & & \mathbb{P}\left(x_{n+1}=-\left(x_{n}+1\right)\right)=p q .
\end{aligned}
$$

Proof. Clearly, $x_{0}=0$. Let $\left.A(n+1,-a):=A\left(\theta_{2(n+1)}-a, \theta_{2(n+1)+1}-a\right)\right)$. In the case $A(n+1,-a)=-I$, we have $\left|u_{n+1,1}(-a)\right|=\left|u_{n, 1}(-a)\right|$, i.e. $x_{n+1}=x_{n}$. If $A(n+1,-a)=$ $\left(\begin{array}{cc}\rho & 0 \\ 0 & 1 / \rho\end{array}\right)$, then $\left|u_{n+1,1}(-a)\right|=\rho\left|u_{n, 1}(-a)\right|$ and $x_{n+1}=x_{n}+1$. Similarly, $A(n+1,-a)=$ $\left(\begin{array}{ll}0 & 1 \\ 1 & 0\end{array}\right)$ implies that $\left|u_{n+1,1}(-a)\right|=\left|u_{n, 2}(-a)\right|=1 /\left|u_{n, 1}(-a)\right|$ and thus $x_{n+1}=-x_{n}$. Finally, $A(n+1,-a)=\left(\begin{array}{cc}0 & -1 / \rho \\ -\rho & 0\end{array}\right)$ gives $\left|u_{n+1,1}(-a)\right|=\frac{1}{\rho}\left|u_{n, 2}(-a)\right|=\frac{1}{\rho\left|u_{n, 1}(-a)\right|}$, i.e. $x_{n+1}=-x_{n}-1$. Thus $x_{n+1}$ is determined by $x_{n}$ and $A(n+1,-a)$. The transition probabilities follow from (9) and (10).

Lemma 4.3. As $n \rightarrow \infty$,

$$
\frac{\mathbb{E}\left(x_{n}^{2}\right)}{n} \rightarrow \frac{q}{2 p}
$$

In particular, we have that for all $n$,

$$
\mathbb{E}\left(\left|x_{n}\right|\right) \leq C n^{1 / 2}
$$

Proof. Let $\alpha=q-p$, we denote by $\mathbb{E}(x \mid y)$ the conditional expectation of $x$ given $y$. It follows that

$$
\mathbb{E}\left(x_{n} \mid x_{n-1}\right)=\alpha^{2} x_{n-1}+q \alpha .
$$

Since $\mathbb{E}\left(x_{n}\right)=\mathbb{E}\left(\mathbb{E}\left(x_{n} \mid x_{n-1}\right)\right)$, we get that

$$
\mathbb{E}\left(x_{n}\right)=\alpha^{2} \mathbb{E}\left(x_{n-1}\right)+q \alpha
$$


and, iterating,

$$
\mathbb{E}\left(x_{n}\right)=\alpha^{2 n} \mathbb{E}\left(x_{0}\right)+q \alpha \frac{1-\alpha^{2 n}}{1-\alpha^{2}} .
$$

Similarly, since

$$
\mathbb{E}\left(x_{n}^{2} \mid x_{n-1}\right)=x_{n-1}^{2}+2 q x_{n-1}+q,
$$

we have that

$$
\mathbb{E}\left(x_{n}^{2}\right)=\mathbb{E}\left(x_{n-1}^{2}\right)+2 q \mathbb{E}\left(x_{n-1}\right)+q .
$$

Another induction gives that, for all $n \geq 3$,

$$
\begin{aligned}
\mathbb{E}\left(x_{n}^{2}\right)= & \mathbb{E}\left(x_{0}^{2}\right)+2 q \frac{1-\alpha^{2 n}}{1-\alpha^{2}} \mathbb{E}\left(x_{0}\right)+n q\left(1+\frac{2 q \alpha}{1-\alpha^{2}}\right) \\
& +2 q^{2} \alpha\left[1-\frac{1}{1-\alpha^{2}}\left(2+\alpha^{4} \frac{1-\alpha^{2(n-2)}}{1-\alpha^{2}}\right)\right] .
\end{aligned}
$$

Since $|\alpha|<1$ and $\mathbb{E}\left(x_{0}\right)=\mathbb{E}\left(x_{0}^{2}\right)=0$ and $q\left(1+\frac{2 q \alpha}{1-\alpha^{2}}\right)=q / 2 p$, we get (15). Using that $\mathbb{E}\left(\left|x_{n}\right|\right) \leq\left(\mathbb{E}\left(x_{n}^{2}\right)\right)^{1 / 2}$, in turn, proves the second assertion and finishes the proof.

Lemma 4.3 is, in fact, a consequence of general extensions of the Central Limit Theorem used in the study of dynamical systems, e.g. Section A.4 of [7]. We include the previous elementary proof for the convenience of the reader.

The main result of this section now follows immediately.

Proof of Theorem 3.1(i). The fact that $\gamma(-a)=0$ follows directly from (12) and Lemma 4.3. The proof of $\gamma(-b)=0$ is identical.

\section{$5 \quad$ Positivity of the Lyapunov Exponent}

In this section we show that, except for the two critical energies discussed above, the Lyapunov exponent (8) is positive. This constitutes the contents of part (iii) of Theorem 3.1 and of Theorem 3.2, For each $\lambda \in \mathbb{T}$, the random variables $\theta_{0}^{\omega}$ and $\theta_{1}^{\omega}$ induce a measure on $G L(2, \mathbb{C})$ through $T\left(\theta_{0}^{\omega}+\lambda, \theta_{1}^{\omega}+\lambda\right)$. Denote the smallest closed subgroup of $G L(2, \mathbb{C})$ generated by the support of this measure by $G_{\lambda, \mu}$. Thus $G_{\lambda, \mu}$ is generated by the matrices $T(\theta, \eta)$, defined in (7), where $\theta$ and $\eta$ vary in $\lambda+\operatorname{supp} \mu$.

Fürstenberg's Theorem [2] states that if $G_{\lambda, \mu}$ is non-compact and strongly irreducible, then

$$
\gamma(\lambda)=\lim _{n \rightarrow \infty} \frac{\mathbb{E}\left(\ln \left\|T_{n}(\omega, \lambda)\right\|\right)}{n}>0 .
$$

The proof that $G_{\lambda, \mu}$ is non-compact for all values of $\lambda \in \mathbb{T}$ was given in [13] and holds for any non-trivial probability distribution $\mu$. For completeness we repeat the proof here.

Lemma 5.1. $G_{\lambda, \mu}$ is non-compact. 
Proof. Let $\theta$ and $\eta$ be on the torus, $\theta \neq \eta$, and let $x:=e^{-i \theta}, z:=e^{-i \eta}$. Let $G(\theta, \eta)$ be the closed group generated by $T(\theta, \theta), T(\eta, \eta), T(\theta, \eta)$, and $T(\eta, \theta)$. Define

$$
\begin{aligned}
& D:=T(\theta, \theta) T(\theta, \eta)^{-1}=\left(\begin{array}{ll}
x \bar{z} & 0 \\
\frac{r}{t}(x \bar{z}-1) & 1
\end{array}\right) \in G(\theta, \eta), \\
& E:=T(\eta, \theta)^{-1} T(\theta, \theta)=\left(\begin{array}{ll}
1 & \frac{r}{t}(1-\bar{x} z) \\
0 & \bar{x} z
\end{array}\right) \in G(\theta, \eta), \\
& L:=D E=\left(\begin{array}{ll}
x \bar{z} & \frac{r}{t}(x \bar{z}-1) \\
\frac{r}{t}(x \bar{z}-1) & \bar{x} z-\frac{r^{2}}{t^{2}}|x \bar{z}-1|^{2}
\end{array}\right) \in G(\theta, \eta), \\
& J:=E D=\left(\begin{array}{ll}
x \bar{z}-\frac{r^{2}}{t^{2}}|\bar{x} z-1|^{2} & \frac{r}{t}(1-\bar{x} z) \\
\frac{r}{t}(1-\bar{x} z) & \bar{x} z
\end{array}\right) \in G(\theta, \eta) .
\end{aligned}
$$

Note that $\operatorname{det} L=\operatorname{det} J=1$ and that $J^{-1}=L^{*}$. Thus we get the self-adjoint element $K:=J^{-1} L$ of $G(\theta, \eta)$. In fact, $K$ is positive definite and $\operatorname{det} K=1$. More calculation shows that

$$
\begin{aligned}
\operatorname{tr} K & =1+\frac{2 r^{2}}{t^{2}}|x \bar{z}-1|^{2}+\left|x \bar{z}-\frac{r^{2}}{t^{2}}\right| x \bar{z}-\left.\left.1\right|^{2}\right|^{2} \\
& =2+\frac{r^{2}}{t^{4}}|x \bar{z}-1|^{4} .
\end{aligned}
$$

As $\theta \neq \eta$ and therefore $x \bar{z} \neq 1$ we conclude that $\operatorname{tr} K>2$. Positivity of $K$ implies that it has an eigenvalue strictly bigger than 1 . Thus, containing all powers of $K$, the group $G(\theta, \eta)$ is non-compact. In particular, with $\theta=\lambda+a$ and $\eta=\lambda+b$, we see that $G_{\lambda, \mu}$ is non compact.

It remains to prove strong irreducibility under the assumptions of Theorem 3.1(iii) as well as under those of Theorem 3.2. Under the already established non-compactness of $G_{\lambda, \mu}$, strong irreducibility of $G_{\lambda, \mu}$ is equivalent to

$$
\#\left\{g \bar{v}: g \in G_{\lambda, \mu}\right\} \geq 3 \text { for all } \bar{v} \in \mathbf{P}\left(\mathbb{C}^{2}\right),
$$

see [2]. We first use this fact to prove that for $\operatorname{supp} \mu=\{a, b\}$ and $|a-b|=\pi,-a$ and $-b$ are the only critical quasi-energies.

Proof of Theorem 3.1(iii). Let $\theta=a+\lambda, \eta=b+\lambda$, with $\lambda \in \mathbb{T} \backslash\{-a,-b\}$. In the terminology introduced above, the condition that $|a-b|=\pi$ can be written as $-x=z \notin$ $\{-1,1\}$. Since $x=-z$, the operator $L \in G_{\lambda, \mu}$ defined by (18) takes the form

$$
L=\left(\begin{array}{cc}
-1 & \frac{-2 r}{t} \\
\frac{-2 r}{t} & -1-\frac{4 r^{2}}{t^{2}}
\end{array}\right)
$$

As $\operatorname{det} L=1$ and $|\operatorname{tr} L|>2, L$ is hyperbolic, hence iterations of $L$ map any direction in $\mathbf{P}\left(\mathbb{C}^{2}\right)$ to infinitely many directions, except when $\bar{v}$ coincides with the direction of one of its eigenvectors, given by $v_{+}=\left(\begin{array}{c}1 \\ \frac{r+1}{t}\end{array}\right), v_{-}=\left(\begin{array}{c}1 \\ \frac{r-1}{t}\end{array}\right)$.

Next we prove that even for the eigenvectors of $L$, we have that $\#\left\{g \bar{v}_{+}: g \in G_{\lambda, \mu}\right\} \geq 3$. 
Under the current conditions, the transfer matrices take the form

$$
\begin{aligned}
& T(\theta, \theta)=\left(\begin{array}{cc}
-x & \frac{r}{t}(1-x) \\
\frac{r}{t}(1-x) & \frac{r^{2}}{t^{2}}(2-x)-\frac{1}{t^{2}} \bar{x}
\end{array}\right), \\
& T(\theta, \eta)=\left(\begin{array}{cc}
x & \frac{r}{t}(-1+x) \\
\frac{r}{t}(1+x) & \frac{r^{2}}{t^{2}} x-\frac{1}{t^{2}} \bar{x}
\end{array}\right), \\
& T(\eta, \theta)=\left(\begin{array}{cc}
-x & \frac{-r}{t}(1+x) \\
\frac{r}{t}(1-x) & \frac{-r^{2}}{t^{2}} x+\frac{1}{t^{2}} \bar{x}
\end{array}\right), \\
& T(\eta, \eta)=\left(\begin{array}{cc}
x & \frac{r}{t}(1+x) \\
\frac{r}{t}(1+x) & \frac{r^{2}}{t^{2}}(2+x)+\frac{1}{t^{2}} \bar{x}
\end{array}\right) .
\end{aligned}
$$

Therefore, we have that

$$
\begin{aligned}
& T(\theta, \theta) v_{+}=\left(\begin{array}{c}
-\frac{r+1}{t^{2}}(r-x) \\
-\frac{r+1}{t^{3}}(r x+\bar{x})+\frac{r(r+1)^{2}}{t^{3}}
\end{array}\right), \\
& T(\theta, \eta) v_{+}=\left(\begin{array}{c}
-\frac{r+1}{t^{2}}(r-x) \\
\frac{r+1}{t^{3}}(r x-\bar{x})+\frac{r}{t}
\end{array}\right), \\
& T(\eta, \theta) v_{+}=\left(\begin{array}{c}
-\frac{r+1}{t^{2}}(r+x) \\
-\frac{r+1}{t^{3}}(r x-\bar{x})+\frac{r}{t}
\end{array}\right) .
\end{aligned}
$$

A simple calculation shows that $\overline{T(\theta, \theta) v_{+}}=\overline{T(\theta, \eta) v_{+}}$only if $x=r$. Similarly, $\overline{T(\theta, \eta) v}=\overline{T(\eta, \theta) v}$ only if $x \in\{-1,1\}$, while assuming that $\overline{T(\theta, \theta) v_{+}}=\overline{T(\eta, \theta) v_{+}}$is equivalent to $(r+1) t^{2}=0$. All these cases are excluded by the assumptions of Theorem 3.1(iii). Therefore, we conclude that $\overline{T(\theta, \theta) v_{+}}, \overline{T(\theta, \eta) v_{+}}$, and $\overline{T(\eta, \theta) v_{+}}$are all different. In a similar way one treats $v_{-}$. We thus have proven that

$$
\#\left\{g \bar{v}: g \in G_{\lambda, \mu}\right\} \geq 3 \text { for all } \bar{v} \in \mathbf{P}\left(\mathbb{C}^{2}\right) .
$$

Combining this with Lemma 5.1, Fürstenberg's Theorem gives the required assertion.

Proof of Theorem 3.2. Again by Fürstenberg's Theorem and Lemma 5.1, proving that $\gamma(\lambda)>0$ for all $\lambda \in \mathbb{C}$ for the case that the support of $\mu$ contains two points of $\mathbb{T}$ that are not diametrically opposed, is reduced to checking condition (20). Each element of the projective space $\mathbf{P}\left(\mathbb{C}^{2}\right)$ is of the form $\bar{v}$ with $v=\left(\begin{array}{l}0 \\ 1\end{array}\right)$ or $v=\left(\begin{array}{l}1 \\ \alpha\end{array}\right)$, for some $\alpha \in \mathbb{C}$. In terms of $x, z$, introduced above, the condition that $|a-b| \notin\{0, \pi\}$ can be written as $x \bar{z} \notin\{-1,1\}$. 
Case I: Let $v=\left(\begin{array}{l}0 \\ 1\end{array}\right)$. The action of the operator $E$, defined in (17), on $\bar{v}$ has the direction of $\left(\begin{array}{c}\frac{r}{t}(x \bar{z}-1) \\ 1\end{array}\right)$, while $E^{2} \bar{v}$ has the direction of $\left(\begin{array}{c}\frac{r}{t}\left((x \bar{z})^{2}-1\right) \\ 1\end{array}\right)$. Thus $\left\{I, E, E^{2}\right\} \subset G_{\lambda, \mu}$ maps $\bar{v}$ into three different elements in $\mathbf{P}\left(\mathbb{C}^{2}\right)$.

Case II: Let $v=\left(\begin{array}{l}1 \\ \alpha\end{array}\right)$, with $\alpha \in \mathbb{C}$. Acting on $\bar{v}$ with the operator $D$ from (16)) results in the direction of $\left(\begin{array}{c}1 \\ \frac{r}{t}(1-\bar{x} z)+\alpha \bar{x} z\end{array}\right)$, while $E \bar{v}$ has the direction of $\left(\begin{array}{c}\frac{1}{\alpha \bar{x} z} \\ 1+\alpha r / t(1-\bar{x} z)\end{array}\right)$.

Defining the map $F$ such that $F: c \mapsto \frac{r}{t}(1-\bar{x} z)+c \bar{x} z$, one sees that $F$ has a single fixed point at $c=r / t$. Since the second iteration $F^{2}: c \mapsto \frac{r}{t}\left(1-(\bar{x} z)^{2}\right)+c(\bar{x} z)^{2}$, has the same value $r / t$ as its only fixed point, we deduce that $\left\{c, F(c), F^{2}(c)\right\}$ are pairwise different except when $c=r / t$. Thus iterations of the operator $D$ take $\bar{v}$ into at least three different directions, unless $\alpha=r / t$.

On the other hand, the map $H: c \mapsto \frac{c \bar{x} z}{1+c r / t(1-\bar{x} z)}$ has fixed points $0,-t / r$, which are also the fixed points of $H^{2}: c \mapsto \frac{c(\bar{x} z)^{2}}{1+c r / t\left(1-(\bar{x} z)^{2}\right)}$. In particular, $\left\{I, E, E^{2}\right\} \subset G_{\lambda, \mu}$ map the direction vector $\left(\begin{array}{c}1 \\ r / t\end{array}\right)$ to three different elements in $\mathbf{P}\left(\mathbb{C}^{2}\right)$. This proves the required condition for strong irreducibility of $G_{\lambda, \mu}$ and once more the result of Lemma 5.1 and Fürstenberg's Theorem finish the proof.

\section{A Unitary Dimer Model}

In this section we study a unitary version of the Dimer model, which is obtained from the Anderson model (6) by doubling up the random phases. More rigorously, let a probability measure $\mu$ on $\mathbb{T}$ be given, define $g: \mathbb{T} \rightarrow \mathbb{T}^{2}$ as $g(\theta)=(\theta, \theta)$ and let $\tilde{\mu}$ be the probability measure supported on the diagonal of $\mathbb{T}^{2}$ induced by $\mu$ through $g: \tilde{\mu}(\tilde{B})=\mu\left(g^{-1}(\tilde{B})\right)$ for Borel sets $\tilde{B}$ in $\mathbb{T}^{2}$. We introduce the probability space $(\tilde{\Omega}, \tilde{\mathcal{F}}, \tilde{\mathbb{P}})$, where $\tilde{\Omega}$ is identified with $\left(\mathbb{T}^{2}\right)^{\mathbb{Z}}, \tilde{\mathcal{F}}$ is the $\sigma$-algebra generated by cylinders of Borel sets in $\mathbb{T}^{2}$ and $\tilde{\mathbb{P}}=\bigotimes_{k \in \mathbb{Z}} \tilde{\mu}$.

For $\omega=\left(\omega_{k}\right)_{k \in \mathbb{Z}} \in \tilde{\Omega}$, the random phases $\theta_{n}^{\omega}, n \in \mathbb{Z}$, used in (4) and (6) to define $U_{\omega}$ are now chosen as

$$
\left(\theta_{2 k}, \theta_{2 k+1}\right)=\omega_{k}, \quad k \in \mathbb{Z}
$$

For this model we again prove that the almost sure absolutely continuous spectrum is empty. We also show that the case of a Bernoulli measure

$$
\mu=p \delta_{a}+q \delta_{b}, \quad p+q=1, \quad a, b \in \mathbb{T}, \quad a \neq b,
$$

gives rise to additional critical quasi-energies, as this is the case of least randomness.

The following theorem states that for any non-trivial distribution $\mu$ on $\mathbb{T}$, i.e. $\{a, b\} \subset$ $\operatorname{supp} \mu$ for $a \neq b$, the Lyapunov exponent is positive for all but a finite set of quasi-energies, given by

$$
M:=\{-a,-b\} \cup\left(M_{a} \cap M_{b}\right),
$$


where

$$
\begin{aligned}
M_{a} & =\left\{\arccos \left(r^{2}\right)-a, 2 \pi-\arccos \left(r^{2}\right)-a, \arccos \left(r^{2}-t^{2}\right)-a, 2 \pi-\arccos \left(r^{2}-t^{2}\right)-a\right\}, \\
M_{b} & =\left\{\arccos \left(r^{2}\right)-b, 2 \pi-\arccos \left(r^{2}\right)-b, \arccos \left(r^{2}-t^{2}\right)-b, 2 \pi-\arccos \left(r^{2}-t^{2}\right)-b\right\} .
\end{aligned}
$$

An immediate consequence is that the almost sure absolutely continuous spectrum of these operators is trivial.

Theorem 6.1. If $\{a, b\} \subset$ supp $\mu$, then for all $\lambda \in \mathbb{T} \backslash M$, the Lyapunov exponent $\gamma(\lambda)$ is strictly positive. In particular, $\Sigma_{a c}=\emptyset$.

As before, we will use Fürstenberg's Theorem [2] to prove positivity of Lyapunov exponents. Let $\widetilde{G}_{\lambda, \mu}$ be the closed group corresponding to $G_{\lambda, \mu}$ from the previous section.

We will show that $\widetilde{G}_{\lambda, \mu}$ is both non-compact and strongly irreducible for all $\lambda$ outside of $M$. As both of these properties carry over to larger groups, we may assume for the rest of the proof of Theorem 6.1 that $\operatorname{supp} \mu=\{a, b\}$. Thus $\widetilde{G}_{\lambda, \mu}$ is generated by just two matrices, $T(\theta, \theta)$ and $T(\eta, \eta)$, where $\theta=a+\lambda, \eta=b+\lambda$.

In order to prove Theorem 6.1, we start by mapping the problem into a somewhat simpler form. In order to simplify the notation, we again let $x:=e^{-i \theta}, z:=e^{-i \eta}$, and let $\rho, \rho_{1}$ be the two eigenvalues of $T(\theta, \theta)$. Since $\operatorname{tr} T(\theta, \theta)=\frac{2 r^{2}}{t^{2}}-\frac{1}{t^{2}}(x+\bar{x})$, and $\operatorname{det} T(\theta, \theta)=$ $\operatorname{det} T(\eta, \eta)=1$, we have the following cases

$$
\left\{\begin{array}{l}
\rho=\overline{\rho_{1}},|\rho|=1, \quad \text { when }|\operatorname{tr} T(\theta, \theta)|<2 \\
\rho=\frac{1}{\rho_{1}}>1, \quad \text { when }|\operatorname{tr} T(\theta, \theta)|>2 \\
\rho=\rho_{1}, \rho^{2}=1, \quad \text { when }|\operatorname{tr} T(\theta, \theta)|=2 .
\end{array}\right.
$$

This allows us to introduce the transformation $N$ given by

$$
N=\left(\begin{array}{cc}
\frac{r}{t}(1-x) & x+\rho \\
x+\rho & -\frac{r}{t}(1-x)
\end{array}\right) .
$$

Using that $(x+\rho)\left(x+\rho_{1}\right)=-\frac{r^{2}}{t^{2}}(1-x)^{2}$, we deduce that $\operatorname{det} N=(x+\rho)\left(\rho_{1}-\rho\right)$. Therefore, $N$ is invertible as long as $|\operatorname{tr} T(\theta, \theta)| \neq 2$. Moreover,

$$
E=N T(\theta, \theta) N^{-1}=\left(\begin{array}{cc}
\rho & 0 \\
0 & 1 / \rho
\end{array}\right)
$$

A short calculation shows that the elements of $F=N T(\eta, \eta) N^{-1}$ are given by

$$
\begin{aligned}
& F_{11}=\frac{1}{t^{2}(\rho-1 / \rho)}\left[2 r^{2}(1+\rho)-(z \bar{x}+\bar{z} x)-\rho(z+\bar{z})\right], \\
& F_{12}=F_{21}=\frac{2 i r}{t^{3}(\rho-1 / \rho)}[\Im(x)-\Im(z)+\Im(z \bar{x})], \\
& F_{22}=\frac{-1}{t^{2}(\rho-1 / \rho)}\left[2 r^{2}\left(1+\frac{1}{\rho}\right)-(z \bar{x}+\bar{z} x)-\frac{1}{\rho}(z+\bar{z})\right] .
\end{aligned}
$$

Notice that since $\theta \neq \eta, F_{12}=0$ if and only if either $\eta=0$ or $\theta=0$.

Since proving non-compactness and strong irreducibility of $\widetilde{G}_{\lambda, \mu}$ is equivalent to proving the same properties for the group $\widetilde{H}_{\lambda, \mu}$ generated by the matrices $E, F$, we will use the latter, somewhat simpler matrices whenever it helps simplifying the proofs. 
Lemma 6.1. For all $\lambda \in \mathbb{T} \backslash\{-a,-b\}$, the group $\widetilde{G}_{\lambda, \mu}$ is non-compact.

Proof. Since $\lambda \neq-a$, we have that $\theta \neq 0$ and thus $\operatorname{tr} T(\theta, \theta) \neq-2$. Therefore, the preceding discussion suggests the proof should be divided into the following cases;

Case I: $\operatorname{tr} T(\theta, \theta)=2$. By (22) we have $\rho=\rho_{1}=1$ and since by definition $T(\theta, \theta) \neq I$ it follows that there exists a non-singular matrix $R$ such that

$$
R T(\theta, \theta) R^{-1}=\left(\begin{array}{cc}
\rho & 1 \\
0 & \rho
\end{array}\right)
$$

Since $\left\|\left[R T(\theta, \theta) R^{-1}\right]^{n}\right\|$ grows with $n$, the group generated by $R T(\theta, \theta) R^{-1}, R T(\eta, \eta) R^{-1}$ is non-compact, which implies that $G_{\lambda, \mu}$ is non-compact.

Case II: $|\operatorname{tr} T(\theta, \theta)|>2$, again by (22),$T(\theta, \theta)$ has an eigenvalue $\rho>1$ which gives the required result.

Case III: $|\operatorname{tr} T(\theta, \theta)|<2$. In this case equations (22), (23) give

$$
E=\left(\begin{array}{cc}
e^{i y} & 0 \\
0 & e^{-i y}
\end{array}\right)
$$

with $y=\arccos \left(\frac{r^{2}}{t^{2}}-\frac{1}{2 t^{2}}(x+\bar{x})\right)$, and $y \in(0, \pi)$. Equations (24) lead to

$$
F=\left(\begin{array}{cc}
\alpha e^{i c} & \beta \\
\beta & \alpha e^{-i c}
\end{array}\right), \quad \alpha \geq 0, \beta \in \mathbb{R}, c \in \mathbb{T} .
$$

Using that $\operatorname{det} F=1$, it follows that $\alpha>0$.

Now we follow a strategy outlined in [10] to show that there exists a sequence of elements in $\widetilde{H}_{\lambda, \mu}$ with unbounded norms. In order to do so, we note that any element of $\mathbf{P}\left(\mathbb{C}^{2}\right)$ can be written in the form

$$
e_{(u, v)}=\left(\begin{array}{c}
e^{i u} \cos (v) \\
e^{-i u} \sin (v)
\end{array}\right), \quad(u, v) \in[0, \pi) \times[0, \pi)
$$

Therefore, for any element $e_{(u, v)}$ of $\mathbf{P}\left(\mathbb{C}^{2}\right)$ we have

$$
F e_{(u, v)}=\left(\begin{array}{l}
\alpha e^{i(c+u)} \cos (v)+\beta e^{-i u} \sin (v) \\
\alpha e^{-i(c+u)} \sin (v)+\beta e^{i u} \cos (v)
\end{array}\right) .
$$

Using that $\alpha^{2}-\beta^{2}=1$, we get

$$
\left\|F e_{(u, v)}\right\|^{2}-1=2 \beta^{2}+4 \alpha \beta \cos (2 u+c) \cos (v) \sin (v) .
$$

Since $\beta=\frac{r}{t^{3}} \frac{\Im(x)-\Im(z)+\Im(z \bar{x})}{\Im(\rho)}$ with distinct $x, z$ and neither equals 1 under the current assumptions, we see that $\beta \neq 0$.

In the case $\beta>0$ : If $\cos (v) \sin (v)=0$, then $\left\|F e_{(u, v)}\right\|^{2}-1>\beta^{2}$ for all $u \in[0, \pi)$. While for $\cos (v) \sin (v)>0,\left\|F e_{(u, v)}\right\|^{2}-1>\beta^{2}$ is equivalent to

$$
\cos (2 u+c)>\frac{-\beta}{4 \alpha \cos (v) \sin (v)}
$$


In particular, the condition $\cos (2 u+c)>\frac{-\beta}{4 \alpha}$ guarantees that $\left\|F e_{(u, v)}\right\|^{2}>1+\beta^{2}$. Defining $K_{+}:=\left\{u \in[0, \pi): \cos (2 u+c)>\frac{-\beta}{4 \alpha}\right\}$, we see that $\left|K_{+}\right|>\pi / 2$ and for all $u \in K_{+}$we have $\left\|F e_{(u, v)}\right\|^{2}>1+\beta^{2}$.

Similarly, for $\cos (v) \sin (v)<0$, let $K_{-}:=\left\{u \in[0, \pi): \cos (2 u+c)<\frac{\beta}{4 \alpha}\right\}$. Then for all $u \in K_{-}$, we have $\left\|F e_{(u, v)}\right\|^{2}>1+\beta^{2}$ and $\left|K_{-}\right|>\pi / 2$.

Hence, given any $v \in[0, \pi)$, there exists an interval $K_{v} \subset[0, \pi)$, i.e. an interval in $\mathbb{R} \backslash \pi \mathbb{Z}$, such that $\left|K_{v}\right|>\pi / 2$ and $\left\|F e_{(u, v)}\right\|^{2}>1+\beta^{2}$, for all $u \in K_{v}$. Therefore, starting with an appropriately chosen vector $e_{(u, v)} \in \mathbf{P}\left(\mathbb{C}^{2}\right)$ such that $\left\|F e_{(u, v)}\right\|^{2}>1+\beta^{2}$, applying $F$ will result in a vector $c e_{\left(u_{1}, v_{1}\right)}$ with $c>1$. Now we apply $E$ as many times as required to get a vector $c e_{\left(\tilde{u}, v_{1}\right)}\left(\right.$ or $\left.-c e_{\left(\tilde{u}, v_{1}\right)}\right)$ such that $\tilde{u} \in K_{v_{1}}$. Iterating this process gives a sequence of vectors with unbounded norms. The case $\beta<0$ is treated similarly. Thus we have proved the non-compactness of $\widetilde{H}_{\lambda, \mu}$, and consequently that of $\widetilde{G}_{\lambda, \mu}$.

The next step is proving that $\widetilde{G}_{\lambda, \mu}$ is strongly irreducible for all $\lambda$ outside the set $M$.

Lemma 6.2. $\widetilde{G}_{\lambda, \mu}$ is strongly irreducible, for all $\lambda \in \mathbb{T} \backslash M$.

Proof. Since we already proved that $\widetilde{G}_{\lambda, \mu}$ is non-compact (Lemma 6.1), it suffices to show that for all $v \in \mathbf{P}\left(\mathbb{C}^{2}\right), \#\left\{g v: g \in \widetilde{G}_{\lambda, \mu}\right\} \geq 3$.

We first note that $\rho^{4}=1$ implies that $|\operatorname{tr} T(\theta, \theta)| \in\{0,2\}$, which in turn implies that $\lambda \in\{-a,-b\} \cup M_{a}$. Therefore, the condition $\lambda \notin\{-a,-b\} \cup M_{a}$ gives that $\rho^{4} \neq 1$. Hence, $\left\{I, E, E^{2}\right\} \subset \widetilde{G}_{\lambda, \mu}$ maps every $v \in \mathbf{P}\left(\mathbb{C}^{2}\right)$ to three different directions unless $v$ coincides with either $\left(\begin{array}{l}1 \\ 0\end{array}\right)$ or $\left(\begin{array}{l}0 \\ 1\end{array}\right)$. At this point, we note that the diagonal elements of the matrix $F$, given in (24), vanish simultaneously only if $\lambda \in\{-a,-b\}$. Thus, iterations of the operator $F$, followed if necessary with iterations of $E$, maps each of the latter directions to at least three different elements of $\mathbf{P}\left(\mathbb{C}^{2}\right)$. This proves strong irreducibility of the group $\widetilde{H}_{\lambda, \mu}$, for all $\lambda \notin\{-a,-b\} \cup M_{a}$, which gives the corresponding result for $\widetilde{G}_{\lambda, \mu}$. A similar argument, replacing the rules of $a$ and $b$, gives a similar assertion for $\lambda \notin\{-a,-b\} \cup M_{b}$, thus finishing the proof.

Proof of Theorem 6.1. The previous two lemmas combined with Fürstenberg's Theorem, immediately give $\gamma(\lambda)>0$ for all $\lambda \in \mathbb{T} \backslash M$. Using Theorem 2.1, we deduce that $\Sigma_{a c}=\emptyset$.

Even though the finiteness of the set $M$ is more than enough to prove the absence of absolutely continuous spectrum for the unitary dimer model, $M$ is by no means optimal. Determining whether or not a certain element of $M$ is, in fact, a critical quasi-energy of $U_{\omega}$ requires further analysis. Nevertheless, the proof suggests that if the support of $\mu$ contains three or more points than generically $M=\emptyset$. Even for the Bernoulli unitary dimer model where $\operatorname{supp} \mu=\{a, b\}$ the analysis is likely to fall into a number of different sub-cases. However, for generic choices of $a, b$ we see that $M_{a} \cap M_{b}=\emptyset$, thus $M=\{-a,-b\}$ and indeed the situation where $\lambda \in\{-a,-b\}$ is readily accessible. Guided by the proof of Theorem 2.2(i) of [11] for the self-adjoint dimer model we prove that,

Proposition 6.1. For a probability measure $\mu$ given by (21), we have the following

(i) If $|a-b| \in \sigma(S)$, then $\gamma(-a)=\gamma(-b)=0$. 
(ii) If $|a-b| \in \rho(S)$, then both $\gamma(-a)>0$ and $\gamma(-b)>0$.

Proof. For $\lambda=-a$, we have that $T(\theta, \theta)=-I$, and $\eta=b-a$. Since $\operatorname{tr} T(\eta, \eta)=$

$\frac{2 r^{2}}{t^{2}}-\frac{2 \cos (\eta)}{t^{2}}$, then $|\operatorname{tr} T(\eta, \eta)| \leq 2$ when $b-a \in \sigma(S)$ and $|\operatorname{tr} T(\eta, \eta)|>2$ if $b-a \in \rho(S)$.

Now, let $m_{n}:=\#\left\{k: 1 \leq k \leq n, \theta_{2 k}^{\omega}=b\right\}$, then $\mathbb{P}$-almost surely

$$
\lim _{n \rightarrow \infty} \frac{m_{n}}{n}=q .
$$

This along with the fact that

$$
\lim _{m_{n} \rightarrow \infty}\left\|[T(\eta, \eta)]^{m_{n}}\right\|^{1 / m_{n}}=\max _{1 \leq i \leq 2}\left|r_{i}\right|,
$$

where $r_{i}$ are the eigenvalues of $T(\eta, \eta)$, gives the results of the proposition.

\section{Continuity of the Lyapunov Exponent}

In this section we prove that, away from the critical points, the Lyapunov exponent is a continuous function of the spectral parameter $\lambda$. The proof of this fact is similar to the one given in [5] for the self-adjoint case.

First, for a compact interval $I$ of quasi-energies with positive Lyapunov exponents, we define the function

$$
\Phi(\lambda, \bar{v})=\mathbb{E}\left(\ln \frac{\left\|T_{\lambda} v\right\|}{\|v\|}\right), \quad \bar{v} \in \mathbf{P}\left(\mathbb{C}^{2}\right), \lambda \in I .
$$

Where $T_{\lambda}:=T\left(\theta_{2 n}^{\omega}(\lambda), \theta_{2 n+1}^{\omega}(\lambda)\right)$ denotes the transfer matrix defined in (7) with the dependance on $\omega$ being suppressed in order to simplify the notation. The next lemma establishes a couple of properties of $\Phi(\lambda, \bar{v})$.

Lemma 7.1. (i) The mapping $\bar{v} \mapsto \Phi(\lambda, \bar{v})$ is continuous on $\mathbf{P}\left(\mathbb{C}^{2}\right)$.

(ii) There exists a constant $C$ such that,

$$
\sup _{\bar{v} \in \mathbf{P}\left(\mathbb{C}^{2}\right)}\left|\Phi(\lambda, \bar{v})-\Phi\left(\lambda_{1}, \bar{v}\right)\right| \leq C\left|e^{i \lambda}-e^{i \lambda_{1}}\right|, \quad \lambda, \lambda_{1} \in I .
$$

Proof. (i) From (7) one sees that the norm of $T_{\lambda}$ is uniformly bounded for all $\lambda, \omega$. Consequently, we also have a uniform bound on $\Phi(\lambda, \bar{v})$. The assertion is then obtained using the dominated convergence theorem.

(ii) First we note that for all $\bar{v} \in \mathbf{P}\left(\mathbb{C}^{2}\right)$,

$$
\left|\Phi(\lambda, \bar{v})-\Phi\left(\lambda_{1}, \bar{v}\right)\right| \leq \mathbb{E}\left(\left|\ln \frac{\left\|T_{\lambda} v\right\|}{\left\|T_{\lambda_{1}} v\right\|}\right|\right) .
$$

Since $\left|\operatorname{det} T_{\lambda} T_{\lambda_{1}}^{-1}\right|=1$, it follows that

$$
\left|\Phi(\lambda, \bar{v})-\Phi\left(\lambda_{1}, \bar{v}\right)\right| \leq \mathbb{E}\left(\ln || T_{\lambda} T_{\lambda_{1}}^{-1}||\right) .
$$

On the other hand, one has

$$
\left\|T_{\lambda} T_{\lambda_{1}}^{-1}\right\| \leq\left\|T_{\lambda_{1}}^{-1}|||| T_{\lambda}-T_{\lambda_{1}}^{-1}\right\|+1 .
$$


Since all norms on $G L(2, \mathbb{C})$ are equivalent, there exists a constant $C_{1}$ such that

$$
\left\|T_{\lambda} T_{\lambda_{1}}^{-1}\right\| \leq C_{1}\left\|T_{\lambda}-T_{\lambda_{1}}^{-1}\right\|_{F}+1
$$

where $\|A\|_{F}^{2} \equiv \sum_{i, j=1}^{2}\left|a_{i j}\right|^{2}$ denotes the Frobenius norm of the matrix $A=\left\{a_{i j}\right\}_{i, j=1}^{2}$. From (17), it is easy to see that ||$T_{\lambda}-T_{\lambda_{1}}^{-1}||_{F} \leq C_{2}\left|e^{i \lambda}-e^{i \lambda_{1}}\right|$. Combining this with (26) and (25) gives the required result.

Before proving the main result of this section, we recall a general fact: If $\mu_{\lambda}$ denotes the probability measure on $G L(2, \mathbb{C})$ induced by $T_{\lambda}$ and $G_{\lambda, \mu}$ is non-compact and strongly irreducible for all $\lambda \in I$, then there exists a unique distribution $\nu_{\lambda}$ on $\mathbf{P}\left(\mathbb{C}^{2}\right)$ that is invariant with respect to $\mu_{\lambda}$. A proof of this fact can be found in [2]. Moreover, we have

Lemma 7.2. For $\lambda \in I$, the mapping $\lambda \mapsto \nu_{\lambda}$ is weakly continuous.

Proof. We start by showing that if the sequence $\left\{\lambda_{n}\right\}_{n \in \mathbb{Z}}$ converges to $\lambda$, then the corresponding measures $\mu_{\lambda_{n}}$ converge weakly to $\mu_{\lambda}$. First recall that for all $\omega \in \Omega, \| T_{\lambda_{n}}(\omega)-$ $T_{\lambda}(\omega)|| \leq d_{n}$, where $d_{n}=C\left|e^{i \lambda_{n}}-e^{i \lambda}\right|$ for some $C \in \mathbb{R}$. Now, let $B \subset G L(2, \mathbb{C})$ such that the boundary of $B$ has zero measure with respect to $\mu_{\lambda}$, i.e. $\mu_{\lambda}(\partial B)=0$. For such a set we have that

$$
\begin{aligned}
\left|\mu_{\lambda_{n}}(B)-\mu_{\lambda}(B)\right| & =\left|\mathbb{P}\left[T_{\lambda_{n}}^{-1}(B)\right]-\mathbb{P}\left[T_{\lambda}^{-1}(B)\right]\right| \\
& \leq \mathbb{P}\left[T_{\lambda_{n}}^{-1}(B) \cap\left(T_{\lambda}^{-1}(B)\right)^{c}\right]+\mathbb{P}\left[T_{\lambda}^{-1}(B) \cap\left(T_{\lambda_{n}}^{-1}(B)\right)^{c}\right]
\end{aligned}
$$

It is not difficult to see that

$$
\begin{aligned}
\mathbb{P}\left[T_{\lambda_{n}}^{-1}(B) \cap\left(T_{\lambda}^{-1}(B)\right)^{c}\right] & \leq \mathbb{P}\left[\left\{\omega \in \Omega: T_{\lambda}(\omega) \in B^{c}, \operatorname{dist}\left(T_{\lambda}(\omega), \partial B\right) \leq d_{n}\right\}\right] \\
& \leq \mu_{\lambda}\left[\left\{A: \operatorname{dist}(A, \partial B) \leq d_{n}\right\}\right]
\end{aligned}
$$

Taking the limit as $n \rightarrow \infty$ and using dominated convergence one sees that

$$
\lim _{n \rightarrow \infty} \mathbb{P}\left[T_{\lambda_{n}}^{-1}(B) \cap\left(T_{\lambda}^{-1}(B)\right)^{c}\right]=\mu_{\lambda}(\partial B)=0 .
$$

Using a similar argument one gets that $\mathbb{P}\left[T_{\lambda}^{-1}(B) \cap\left(T_{\lambda_{n}}^{-1}(B)\right)^{c}\right] \rightarrow 0$ as $n \rightarrow \infty$. Therefore, we have

$$
\lim _{n \rightarrow \infty} \mu_{\lambda_{n}}(B)=\mu_{\lambda}(B)
$$

Since this is true for any set $B$ with $\mu_{\lambda}(\partial B)=0$, weak convergence of $\mu_{\lambda_{n}}$ to $\mu_{\lambda}$ follows [20]. In order to get the weak convergence of $\nu_{\lambda_{n}}$, we use the fact that the set of invariant measures on $\mathbf{P}\left(\mathbb{C}^{2}\right)$ is compact in the weak* topology [20], thus every subsequence of $\left\{\nu_{\lambda_{n}}\right\}$ has a weakly convergent subsequence and since the limit of each of those subsequences is invariant with respect to $\mu_{\lambda}$ it equals $\nu_{\lambda}$ by uniqueness of the latter. A short contradiction argument shows that $\nu_{\lambda_{n}}$ has to converge weakly to $\nu_{\lambda}$.

The Lyapunov exponent can be expressed in terms of the mapping $\Phi$ and the measure $\nu_{\lambda}$ as

$$
\gamma(\lambda)=\int \Phi(\lambda, \bar{v}) d \nu_{\lambda}(\bar{v})
$$

Now we are ready to prove that for any interval $I$ for which $G_{\lambda, \mu}$ is non-compact and strongly irreducible for all $\lambda \in I$, we have 
Theorem 7.1. The Lyapunov exponent $\gamma(\lambda)$ is a continuous function of $\lambda \in I$.

Proof. Let $\lambda \in I$ and choose $\left\{\lambda_{n}\right\} \subset I$ a sequence of quasi-energies such that $\lambda_{n} \rightarrow \lambda$ as $n \rightarrow \infty$. It follows that

$$
\begin{aligned}
\lim _{n \rightarrow \infty} \gamma\left(\lambda_{n}\right) & =\lim _{n \rightarrow \infty} \int \Phi\left(\lambda_{n}, \bar{v}\right) d \nu_{\lambda_{n}}(\bar{v}) \\
& =\lim _{n \rightarrow \infty}\left[\int \Phi(\lambda, \bar{v}) d \nu_{\lambda_{n}}(\bar{v})+\int\left(\Phi\left(\lambda_{n}, \bar{v}\right)-\Phi(\lambda, \bar{v})\right) d \nu_{\lambda_{n}}(\bar{v})\right] \\
& =\int \Phi(\lambda, \bar{v}) d \nu_{\lambda}(\bar{v})
\end{aligned}
$$

with the last equality following from Lemma 7.2 along with part (ii) of Lemma 7.1 .

Acknowledgements: Partial financial support for this project was provided through NSF grant DMS-0245210. We would like to thank Alain Joye for useful discussions and Dirk Hundertmark for providing the simple proof of Lemma 4.3, E. H. also acknowledges support through a Junior Research Fellowship at the Erwin Schrödinger Institute in Vienna, where part of this work was done.

\section{References}

[1] Bellissard, J., "Stability and instability in quantum mechanics", Trends and Developments in the Eighties, 1-106, World Sci. Publishing, Singapore, 1985.

[2] Bougerol, P. and Lacroix, J.: Products of random matrices with applications to Schrödinger operators, Progress in Probability and Statistics, Vol. 8, Birkhäuser, Boston, MA, 1985.

[3] Blatter, G. and Browne, D., "Zener tunneling and localization in small conducting rings", Phys. Rev. B 37, 3856 (1988).

[4] Bourget, O., Howland, J. S. and Joye, A., "Spectral Analysis of Unitary Band Matrices", Commun. Math. Phys. 234, 191-227 (2003).

[5] Carmona, R. and Lacroix, J.: Spectral theory of random Schrödinger operators, Probability and its Applications, Birkhäuser, Boston, MA, 1990.

[6] Cantero, M. J., Moral, L. and Velázquez, L., "Five-Diagonal Matrices and Zeros of Orthogonal Polynomials on the Unit Circle", Linear Algebra Appl. 362, 29-56 (2003).

[7] Chernov, N. and Dolgopyat, D., "Brownian Brownian Motion - I", Memoirs Amer. Math. Soc., to appear.

[8] Carmona, R., Klein, A. and Martinelli, F., "Anderson localization for Bernoulli and other singular potentials", Comm. Math. Phys. 108, 41-66 (1987).

[9] Combescure, M.: Recurrent versus diffusive quantum behavior for time dependent Hamiltonians, Oper. Theory Adv. Appl. 57, Birkhäuser, Basel, 1992.

[10] Damanik, D., Sims, R. and Stolz, G., "Localization for one-dimensional, continuum, Bernoulli-Anderson models", Duke Math. J. 114, 59-100 (2002).

[11] De Bièvre, S. and Germinet, F., "Dynamical localization for the random dimer Schrödinger operator", J. Statist. Phys. 98, 1135-1148 (2000). 
[12] Dunlap, D. H., Phillips P. W. and Wu, H.-L., "Absence of localization in a randomdimer model", Phys. Rev. Lett., 65, 88-91 (1990).

[13] Hamza, E., Joye, A. and Stolz, G., "Localization for random unitary operators", Lett. Math. Phys. 75, 255-272 (2006).

[14] Howland, J. S., "Quantum stability", Schrödinger operators , Lecture Notes in Phys., 403, 100-122, Springer, Berlin, 1992.

[15] Jitomirskya, S., Schulz-Baldes, H. and Stolz, G., "Delocalization in random polymer models" Comm. Math. Phys., 233, 27-48 (2003).

[16] Joye, A., "Density of States and Thouless Formula for Random Unitary Band Matrices", Ann. Henri Poincaré 5, 347-379 (2004).

[17] Lenz, D., private communication

[18] Joye, A., "Fractional Moment Estimates for Random Unitary Band Matrices", Lett. Math. Phys. 72, 51-64 (2005).

[19] Simon, B.: Orthogonal Polynomials on the Unit Circle, AMS Colloquium Publications, Vols. 54.1 and 54.2, American Mathematical Society, Providence, RI, 2005.

[20] Walters, P.: An introduction to ergodic theory, Graduate Texts in Mathematics, Vol. 79, Springer-Verlag, New York, 1982. 